\title{
Avalanche! Reanimating Multiple Roles in Child Computer Interaction Design
}

\author{
Timo Göttel \\ University of Hamburg, Department of Informatics, Vogt-Kölln-Str. 30, \\ 22527 Hamburg, Germany \\ tgoettel@acm.org
}

\begin{abstract}
This paper values children as design partners in Child Computer Interaction to ensure building environments highly suitable for the target group. However, it appears beneficial to address underlying roles, align design processes on school activities or give considerations to the voluntary nature of such projects. We conducted eight projects with 75 pupils using initial learning environments. We found that starting design processes with pupils as users helps to identify further design steps that can be consequently tackled with the former users as informants and design partners. Thus, we suggest the Avalanche Design Cycle to engage children as fresh users at several stages asking them to become informants and design partners consecutively. The Avalanche Design Cycle is an iterative design process complying with school life allowing pupils to join/leave design teams. Thereby, it is aiming at sustained team sizes and more representative groups.
\end{abstract}

Keywords: Child-centered design, users, informants, design partners.

\section{Introduction}

Child Computer Interaction (CCI) is a subcategory of Human Computer Interaction with constant growth over the last decade ${ }^{1}$. Development of computer environments for children needs specific design methodologies because adult designers often hold misconceptions of child users based on fading individual childhood memories [2]. Additionally, children have specific requirements that for example cannot be associated with the goal to complete a task as it is done when designing for adult users [2]. Because of these profound differences current CCI research is focused on engaging children in design processes and accordant success stories. In this paper we highlight the need to open design processes for external user tests with children. Thus, we examine current CCI design methodologies based on a literature review and project observations to illustrate shortcomings in using these methodologies. Consequently,

\footnotetext{
${ }^{1}$ In the past eleven years, the CCI community has established the annual Conference on Interaction Design and Children (IDC), an IFIP TC13 group, and is "featured community" at the CHI Conference on Human Factors in Computing Systems for the 3rd time in 2013.
} 
we present a prototyped design process allowing design teams to provide entry points, explicitly address roles, and consider school life.

\section{Related Work}

Harel [5] first presented the idea of children designers. She enabled children to design learning environments on given educational objectives for younger children using a computer. Consequentially, Scaife et al. [13] pointed out that designing for or with children is an essential question. In this regard, Scaife et al. propose a hybrid form of User-Centered Design (UCD) and Participatory Design (PD) to respond to child characteristics. Yet, they identify issues in seeing children as adequate design partners because children cannot define their own learning goals. Therefore, Scaife et al. understand children as informants testing prototypes, verbalizing needs or ideas, and designing through scenarios or games.

Later Druin [3] reviewed PD with children and presents four roles to involve children in design (Figure 1): Users are observed while utilizing technology to get an understanding on how to improve it. Testers are requested to comment on prototypes while using them. Informants are involved at the design process at certain stages. Informants contextually switch roles between user and tester. Design partners are full members of a design team with adults. Druin does not see a universal role for every context but she states that these roles are building upon each other. In this connection, many reports on successful implementations of children as design partners appeared in recent years (e.g. [8, 12]).

\section{The Child as...}

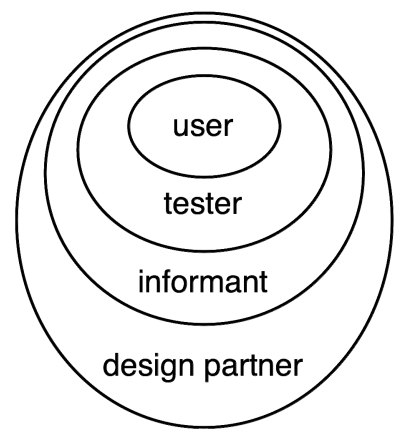

Fig. 1. Design roles according to Druin [3]

Accordingly, a recent review on CCI research acknowledges a highly regarded value in relying on design partners amongst the research community. Yet, the paper also reveals that in contradiction to these statements most papers presenting a system relied on testers to inform the design (59\%) whereas only $31 \%$ were build upon projects with design partners [14]. This is surprising because design partners can theoretically be addressed as informants. The mismatch may be explained by a lack of 
reports in CCI literature on how to activate multiple roles with design partners. This may be in fact a large barrier considering that even experienced adult designers need strong and comprehensive tools as for example Personas to impersonate conventional users for evaluation sessions [1]. Accordingly, CCI researchers may need a model that allows to activate multiple roles when designing with design partners because they do not want to miss the chance to benefit from the other roles (e.g. informants).

When looking at CCI literature, iterative evaluations with external users are rarely presented. This is astonishing because UCD for example strongly relies on external testers throughout the entire process. An article by Kelly et al. [7] is a good example for CCI papers that present user tests with pupils without prior knowledge of the system or the design process: The evaluations mark the end of their project. Kelly et al. state that "the evaluation produced useful results for improvements, which were completely met at the second evaluation stage". This suggests that the tested environments can be seen as high-fidelity prototypes. Yet, literature shows that evaluating low-fi prototypes with external users during the whole design processes can provide more insights than revealing minor usability issues at the end of a project (cp. [10]). Hence, current CCI design processes do not fully consider user tests with fresh users as recurring milestones. Thereby they miss the chance to share prototypes with external users to establish feedback loops that may produce more universal results.

In a different context, Resnick [11] promotes a "creative thinking spiral" that highlights the possibility to share preliminary outcomes with others as an essential component in technology that supports learning and user engagement (Figure 2). We confirm such requests by adding that sharing should also be present in design processes to facilitate a coherent experience for pupils. In fact, many CCI processes rely on sharing ideas amongst team members but fall short in sharing prototypes with externals (e.g. [8]). Hence, we want to expand sharing in CCI processes and understand this as a good motivation for recurring user tests in project layouts.

Additionally, some authors mention that the findings of projects with design partners are hard to transfer to other contexts because of rather specific and small sample design teams [12, 8]. In this connection, Moraveji et al. [9] present many contradicting results in literature on PD with children. They ascribe that to processes of "finding" qualified children raising the question: Does selecting the "right" participants really provide representative groups?

Furthermore, while many projects are conducted in school settings (e.g. [7, 13]), CCI literature hardly describes the integration of design projects in school environments or obligations of the child participants. Only Horton et al. [6] acknowledge that sound descriptions and methodologies promoting the course of action are needed to help pupils, teachers, and researchers building sustainable relationships by choice.

In summary, we understand design partners that produce good results. Yet, we argue that most CCI environments are build with informants because with design partners it is hard to benefit from insightful findings usually provided by the underlying roles of design partners mentioned by Druin [3]. Hence, we want to bring forward a model that understands design partners as a consecutive process from users to informants to (experienced) design partners. Furthermore, we reason that sharing with externals as proposed in the "creative thinking spiral" is often neglected during CCI 
design processes. Hence, we see a strong need to establish design processes that build upon multiple roles and can be embedded in school contexts. Thereby, we want to establish a design process that is constantly refreshed by new team members to ensure sustained team sizes in unsolicited projects and assemble representative groups.

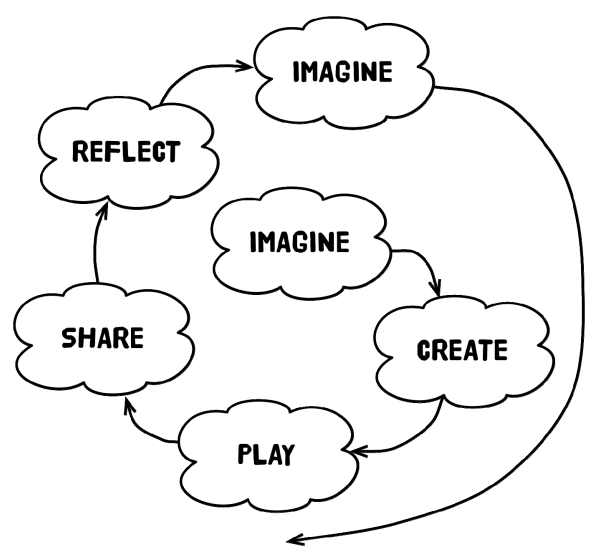

Fig. 2. "Creative thinking spiral" according to Resnick [11]

\section{Project Observations}

In research on the Virtual Sandbox [4], we conducted seven projects using initial learning environments (ILEs ${ }^{2}$ ) together with a total of 75 pupils (age 10-17). The participants were asked to design interactive stories acting as users on an ILE. During the projects external pupils playtested the scenarios. It helped the team members to get new perspectives and ideas. Moreover, it allowed the testers to become interested in the project and get into contact with the experienced designers. Although we did not find such remarks in CCI literature, we became aware that working with pupils on a voluntary basis often comes with uncertainties. Although the projects generally lasted only few days (1-5 days) we encountered pupils who simply did not show up after some sessions, or others who wanted to join later.

The arrangement of the projects showed that pupils have different motivation or engagement levels during a school year. For example, test periods or some days prior to vacations can hardly be used to host intense design sessions. More likely, such times were used to play around or relax. It is stunning that established CCI methodologies do not reflect in detail on everyday life of pupils that turns out to be strongly taken by school and leisure activities. Hence, to some extent this everyday life is competing against additional design projects conducted by the CCI community. The article by Horton et al. [6] confirms this point of view.

\footnotetext{
${ }^{2}$ For definition and instances of ILEs refer to correspondent special issue of ACM Transactions on Computing Education 10, 4 (November 2010).
} 
To study role switching processes we asked 12 former users in a following eighth project to comment as informants on our observations on issues with ILEs when using them in teams. After an extensive discussion, we successfully engaged them as design partners in four sessions that were focused on supporting group work in ILEs applying brainstorming techniques, paper prototypes, and a variant of comicboarding (cp. [9]). Switching roles from users to informants to design partners appeared to be natural to the pupils. They were able to build their arguments on previous user sessions.

One topic clearly showed issues when designing with pupils. When asked how to document scripts for other team members they did not see sense in discussing documentation features at all. They were sure that everyone would understand their scripts. Furthermore, they did not consider approaches presented by adult team members. In such a situation introducing new test users to share current outcomes may highlight group thinking issues. Consequently, observing their peers instead of discussing with senior designers may help child designers to rethink their design or approach.

In conclusion, engaging child users as informants and design partners afterwards provided a wide range of approaches and ways of thinking not influenced by prior design decisions. Nevertheless, based on our experiences and concerns of Scaife et al. [13] we argue that design partners do accurately support developing environments but cannot sufficiently assist in identifying important following steps. In spite of compliance with the idea of design partners, such situations require input by experienced (adult) designers based on shared observations available to all team members. Accordingly, we claim for a clear process that addresses users, informants and design partners at different stages of the design process. Furthermore, we see a need for aligning such approaches with school activities and offer chances to join or leave design projects.

\section{Avalanche Design Cycle}

Project observations showed that pupils find it easy to switch roles when offered clear reference points during design processes. Therefore, we argue that CCI design processes should be arranged in iterative phases including pupils in four consecutive steps as users, informants, design partners, and experienced design partners. Users allow researchers and designers to observe the actual usage of environments without prompting the users to evaluate their activities. Consequential, hypotheses can be developed and discussed with the users afterwards allowing them to switch from users to informants. This transition from user to informant offers many insights and reflections allowing to omit the tester role presented by Druin [3]. In a following step, the former informants should be encouraged to support the upcoming design process as design partners. Assuring an iterative character milestones should be arranged to get new users involved in evaluation sessions. New users should then be encouraged to join the existing design team to become informants and design partners themselves. Introducing new users is important because they provide new unbiased perspectives and represent various approaches in design and implementation. Fresh team members should be tutored by experienced design partners. Based on the results of Harel [5], it 
is feasible to provide entry points during a design process allowing experienced pupils to tutor new design partners.

To meet these claims, we propose the Avalanche Design Cycle (ADC) which is incremental and open for new participants as drafted in Figure 3. It consists of recurring user tests to share outcomes with pupils who do not have prior knowledge on the system or the design process. After these stages the users are asked to become informants to discuss their ideas on the used environment and to suggest design ideas. Furthermore, they are encouraged to comment on observations and implications of the user tests and join the team as design partners. Thus, user tests enable design teams to set or shift focuses according to observations on user behaviors and provide opportunities to reflect on one's own activities. Additionally, the user test stages represent sound opportunities to motivate new pupils to become involved in a design process.

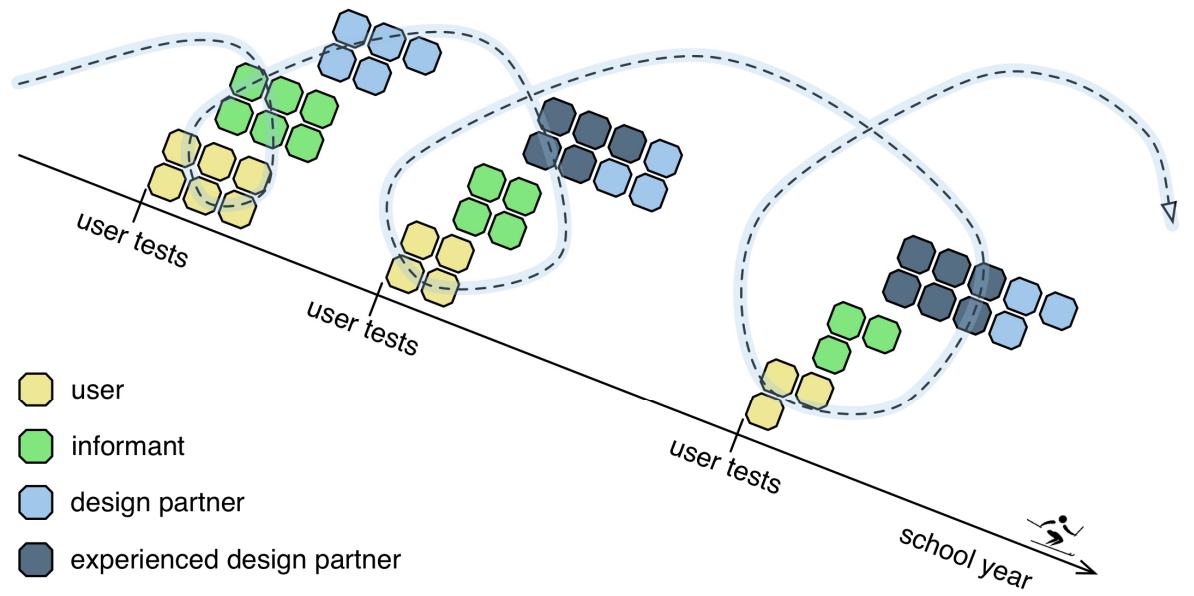

Fig. 3. The Avalanche Design Cycle (ADC) aiming at a sustained growth of design teams by offering recurring new incentives. User tests provide entry/exit points for pupils and can be aligned to schools' test periods offering playful aspects with minimal time and effort for pupils during these crunchtimes.

From our point of view, ADC should initially start with user tests on prebuilt prototypes or commercial systems. It helps building a common ground and allows pupils to influence further research directions by switching roles thereafter. After the initial user test all upcoming evaluations should be done on prototypes build by the design team.

An ADC process engages former users in succeeding sessions by asking them to comment on observations of the design team. This extensive discussion supports users in realizing that they actually could contribute design ideas build upon their experiences as users. This marks the point to ask the informants if they are interested in becoming a part of the design team. We suggest to apply brainstorming techniques, paper prototyping and further variants of PD that can be found in CCI literature (e.g. $[7,8,9,12])$. 
For design partners it is important to observe their peers in using the prototypes. These insights help to rethink design approaches and to support experienced design partners in tutoring informants or freshly recruited design partners.

Additionally, ADC allows to conduct long term and large scale-projects fitting into dynamic scenarios at school life: By constantly introducing new team members during user tests a sustained team size is guaranteed. User tests mark a point in time that allows former members to conveniently leave a team at the end of a school year or when focusing on something else. Consequently, the concerns of Moraveji et al. [9] could be addressed because constantly attracting new users who can become design partners prevents from selecting only the "right" participants. Thus, it would give access to a wide range of pupils to act as users and let them decide afterwards if they want to become involved in the design of the respective system. Besides, school life has predefined times building on strong commitment by pupils. As we understand user tests they should be playful and fun with minimal time and effort for pupils. Thus, user tests should be aligned with known crunchtimes to keep on track in the design process while offering compensation to pupils during these times.

\section{Discussion}

In this paper we highlight a need for multiple consecutive roles in CCI design processes based on literature review and project observations implementing established CCI methodologies and roles. Yet, we derived ADC as a premise to identify issues, explicitly address roles, and rethink design processes with children as well as considering surrounding settings. In presenting ADC, we want to encourage other community members to reflect on their activities and connect it with ADC. Hence, we promote ADC as an ongoing "open source" prototyping process rather than being a final methodology. To open up this process, we put forward hypotheses on ADC that we hope will be challenged from different CCI community perspectives - in addition to our own research agenda on evaluating and refining the Avalanche Design Cycle:

ADC may offer...

- lightweight project takeoffs by engaging children as users

- several entry/exit points to consider unsolicited project layouts

- recurrent milestones to share outcomes of design teams with externals

- activation of insightful design roles (users, informants, design partners)

- alignment with school events and teachers projects

- constant/growing design teams by constantly introducing fresh users

Accordingly, ADC has to be comprehensively implemented and evaluated in long term studies at schools. Further adjustments are expected in alignment to school life and the transitions from user to informant to (experienced) design partner. Consequently, more concrete activities supporting the role transitions and interactions should emerge. Additionally, observations on a broad implementation of ADC should give insights on how to engage teachers, principles, and IT administrators to foster a smooth, fun, and engaging design cycle fully embedded in school life. 


\section{References}

1. Adlin, T., Pruitt, J.: The Essential Persona Lifecycle: Your Guide to Building and Using Personas. Morgan Kaufmann (2010)

2. Antle, A.N.: Child-Based Personas: Need, Ability and Experience. Cognition, Technology and Work 10(2), 155-166 (2008)

3. Druin, A.: The Role of Children in the Design of New Technology. Behaviour \& Information Technology 21(1), 1-25 (2002)

4. Göttel, T.: Virtual Sandbox: Adding Groupware Abilities to Scratch. In: 8th International Conference on Interaction Design and Children, pp. 158-161. ACM, New York (2009)

5. Harel, I.: Children Designers: Interdisciplinary Constructions for Learning and Knowing Mathematics in a Computer-Rich School. Ablex Publishing (1991)

6. Horton, M., Read, J.C., Mazzone, E., Sim, G., Fitton, F.: School Friendly Participatory Research Activities with Children. In: CHI 2012 Extended Abstracts on Human Factors in Computing Systems, pp. 2099-2104. ACM, New York (2012)

7. Kelly, S.R., Mazzone, E., Horton, M., Read, J.C.: Bluebells: a Design Method for ChildCentred Product Development. In: 4th Nordic Conference on Human-Computer Interaction, pp. 361-368. ACM, New York (2006)

8. Laughnan, J.: The Organization of Inventing and Prototyping Activities with Children as Design Partners. In: 2004 Conference on Interaction Design and Children, pp. 127-128. ACM, New York (2004)

9. Moraveji, N., Li, J., Ding, J., O'Kelley, P., Woolf, S.: Comicboarding: Using Comics as Proxies for Participatory Design with Children. In: SIGCHI Conference on Human Factors in Computing Systems, pp. 1371-1374. ACM, New York (2007)

10. Nielsen, J.: Paper Versus Computer Implementations as Mockup Scenarios for Heuristic Evaluation. In: IFIP TC13 3rd International Conference on Human-Computer Interaction, pp. 315-320. North-Holland Publishing Co., Amsterdam (1990)

11. Resnick, M.: Sowing the Seeds for a More Creative Society. Learning \& Leading with Technology, 18-22 (2007)

12. Roussou, M., Kavalieratou, E., Doulgeridis, M.: Children Designers in the Museum: Applying Participatory Design for the Development of an Art Education Program. In: 6th International Conference on Interaction Design and Children, pp. 77-80. ACM, New York (2007)

13. Scaife, M., Rogers, Y., Aldrich, F., Davies, M.: Designing for or Designing With? Informant Design for Interactive Learning Environments. In: SIGCHI Conference on Human Factors in Computing Systems, pp. 343-350. ACM, New York (1997)

14. Yarosh, S., Radu, I., Hunter, S., Rosenbaum, E.: Examining Values: an Analysis of Nine Years of IDC Research. In: 10th International Conference on Interaction Design and Children, pp. 136-144. ACM, New York (2011) 